\title{
Tracing the location of powdery mildew resistance-related gene Stpk- $V$ by FISH with a TAC clone in Triticum aestivum-Haynaldia villosa alien chromosome lines
}

\author{
YANG XueMing ${ }^{1,2}$, CAO AiZhong ${ }^{1}$, SUN YuLei ${ }^{1} \&$ CHEN PeiDu ${ }^{1 *}$ \\ ${ }^{1}$ State Key Laboratory of Crop Genetics and Germplasm Enhancement, Cytogenetics Institute, Nanjing Agricultural University, Nanjing 210095 , \\ China; \\ ${ }^{2}$ Provincial Key Laboratory of Agrobiology, Institute of Biotechnology, Jiangsu Academy of Agricultural Sciences, Nanjing 210014, China
}

Received November 9, 2012; accepted January 23, 2013; published online July 22, 2013

\begin{abstract}
Bacterial artificial chromosomes (BACs) or yeast artificial chromosomes (YACs) containing large inserts as probes for fluorescence in situ hybridization (FISH) have been used in the physical mapping of specific DNA sequences, especially for single- or low-copy sequences. Our earlier study identified $S t p k-V$, a powdery mildew resistance-related gene located on the 6VS chromosome arm of the wild grass Haynaldia villosa (tribe Triticeae), and obtained several Triticum aestivum-H. villosa alien chromosome lines carrying the $S t p k-V$ gene. However, the precise physical location of the Stpk-V gene on chromosome 6VS is not known. In this study, we used TAC-FISH with TAC15 as the probe coupled with sequential genomic in situ hybridization (GISH) to determine the physical location of the Stpk-V gene in different T. aestivum-H. villosa 6V alien chromosome lines, including addition, substitution and translocation lines. The result indicated that the fraction length of the Stpk-V locus is $0.575 \pm 0.035$ on the $6 \mathrm{~V}$ chromosome short arm and this was confirmed by FISH using TAC15 as the probe for tracing the $S t p k-V$ gene in other genetic stocks. The cytological mapping strategies used in this study will be of benefit for tracing the alien gene location in the course of introducing desirable traits from wild species.
\end{abstract}

fluorescence in situ hybridization (FISH), transformation-competent artificial chromosome (TAC), serine/threonine protein kinase gene from the V-genome (Stpk-V), Triticum aestivum-Haynaldia villosa alien chromosome lines

Citation: $\quad$ Yang X M, Cao A Z, Sun Y L, et al. Tracing the location of powdery mildew resistance-related gene Stpk-V by FISH with a TAC clone in Triticum aestivum-Haynaldia villosa alien chromosome lines. Chin Sci Bull, 2013, 58: 4084-4091, doi: 10.1007/s11434-013-5851-x

Fluorescence in situ hybridization (FISH) has been widely used for mapping repetitive DNA sequences and multi-copy gene families [1]. Genomic in situ hybridization (GISH), a modification of FISH using total genomic DNA from one species as the probe, is a powerful tool for the detection of introgressed alien chromatins [2]. In the tribe Triticeae, localized repetitive sequences are useful as cytogenetic markers for chromosome identification and GISH has been used for detecting alien chromosomes in wheat (Triticum aestivum) alien chromosomal lines $[3,4]$. The physical mapping of single- or low-copy sequences on metaphase chromosomes is difficult but necessary for gene or marker localization in wheat.

*Corresponding author (email: pdchen@njau.edu.cn)
The use of genomic DNA fragments in large-insert vectors, such as bacterial artificial chromosomes (BACs) or yeast artificial chromosomes (YACs), in combination with FISH (BAC-FISH or YAC-FISH) has been exploited for mapping genes in some plant species with small genomes, including rice (Oryza sativa) [5-9], sorghum (Sorghum sp.) [10], Ipomoea trifida [11] and Beta vulgaris [12]. It is difficult to generate unique locus-specific FISH signals from most wheat BACs because the wheat genome contains a huge content of repetitive DNA sequences (tandem repeats, transposable elements) scattered throughout the genome [3]. In order to physically map a useful gene Stpk-V from the wild grass Haynaldia villosa (Dasypyrum villosum, $2 n=2 x=$ 14 , genome VV), a positive transformation-competent artificial chromosome (TAC) clone containing the gene was 
used as the probe for FISH to try to determine the exact physical location of the gene in this study.

Powdery mildew (PM), caused by Blumeria graminis $\mathrm{f}$. sp. tritici, is one of the major diseases of wheat worldwide. Pm21, a gene located in the 6VS chromosome bin (fragment length $0.00-0.58$ ) of $H$. villosa, confers a high level of broad-spectrum resistance to $B$. graminis [13,14]. A serine/threonine kinase gene Stpk-V of $H$. villosa, which is up-regulated by $B$. graminis inoculation, was isolated using microarray in our earlier work, and the sequence-specific PCR marker CINAU15, which is linked to Pm21, was designed on the basis of the sequence of the Stpk- $V$ gene [15]. The location of the $S t p k-V$ gene was located on the 6VS chromosome by PCR analysis in materials containing 6VS, and further mapped to the chromosome bin FL $0.45-0.58$ by using a resistant alien deletion line del6VS-1 (FL 0.58) and a susceptible alien deletion line del6VS-2 (FL 0.45) [15]. TAC15 and a 5160-bp sub-clone containing the whole genomic sequence of $S t p k-V$ were obtained by screening a TAC library of T6VS/6AL with the gene-based marker CINAU15 [16,17]. Sequence comparisons showed that the cDNA of Stpk- $V$ matches the exons of the TAC sub-clone completely. Therefore, TAC15 was suitable for physical mapping of the Stpk- $V$ gene. A TAC-FISH signal was detected near the telomeres of 6VS in del6VS-1, and the signal was located from FL 0.45 to 0.58 of $6 \mathrm{VS}$ in the T. aestivum-H. villosa translocation line T6VS·6AL [18]. Until now, the precise physical location of this gene was not known. Owing to the low frequency of pairing and suppressed recombination between the 6VS of $H$. villosa and 6AS of $T$. aestivum, it is extremely difficult to characterize the Stpk-V locus through meiosis crossover and recombination.

To obtain more accurate physical mapping information of the Stpk- $V$ gene and facilitate exploitation of the new resistance-related gene in the adjacent area of $S t p k-V$ loci, this study used a sensitive FISH and sequential GISH in different $T$. aestivum $-H$. villosa alien chromosome lines, including the $6 \mathrm{~V}$ addition line, the $6 \mathrm{~V}(6 \mathrm{~A})$ substitution line, the 6VS/6AL translocation lines, the 6VS deletion lines and translocation lines with the small 6VS chromosome segment. The genotypes susceptible to powdery mildew, which contain the $6 \mathrm{~V}$ chromosome segment, were also used to determine the location of the $S t p k-V$ gene. The results derived from sequential TAC-FISH and GISH methods provide reliable information for the precise physical location of the $S t p k-V$ gene, and are helpful for tracing the $S t p k-V$ gene using the cytogenetic method in different genetic stocks.

\section{Materials and methods}

\subsection{Plant materials}

H. villosa, Triticum durum cv. 1286-H. villosa amphiploid (AABBVV), T. aestivum-H. villosa 6V addition line 06R33, $6 \mathrm{~V}$ (6A) substitution line 06R41, 6VS/6AL translocation lines 92R90 and 92R137 and its derivative variety Nannong 9918, deletion addition line del6VS-1 lacking the distal $42 \%$ of the short arm of chromosome $6 \mathrm{~V}$, 6VS small segment translocation lines YC72-2 and YC138-4 with the 6VS chromosome bin at FL0.45-0.58 and 0.35-1.00, respectively, were resistant to powdery mildew. Deletion addition line del6VS-2, which lacks the distal $55 \%$ of the short arm of chromosome $6 \mathrm{~V}$, the $6 \mathrm{VS}$ small segment translocation lines HY86-1 and HY158-5, which contain the 6VS chromosome bin at FL0.00-0.45 and 0.70-1.00, respectively, were susceptible to powdery mildew. These materials were used for physical mapping of the Pm21 locus. The H. villosa accession used in this study was kindly provided by the Cambridge Botanical Garden, UK. The T. aestivum-H. villosa $6 \mathrm{~V}$ alien chromosome lines were developed and provided by the Cytogenetics Institute, Nanjing Agricultural University (CINAU) [13,14,19-23], where T. durum cv. 1286, and $T$. aestivum cv. Chinese Spring were maintained.

\subsection{Molecular marker verification}

Plant materials used for TAC-FISH were tested using the molecular marker CINAU15, which is specific to the Stpk-V gene. Genomic DNA was isolated by the CTAB method [24]. PCR was performed as described [15]. The PCR products were electrophoresed in non-denaturing $8 \%(\mathrm{w} / \mathrm{v})$ polyacrylamide gels.

\subsection{Chromosome preparation}

Seeds were left to germinate on moist filter paper for $24 \mathrm{~h}$ at $22^{\circ} \mathrm{C}$, kept for $24 \mathrm{~h}$ at $4{ }^{\circ} \mathrm{C}$ followed by $24 \mathrm{~h}$ at $22^{\circ} \mathrm{C}$. Root tips $\sim 1.5 \mathrm{~cm}$ long were harvested, kept in ice-cold water for $24 \mathrm{~h}$ before fixation in $3: 1(\mathrm{v} / \mathrm{v})$ ethanol/acetic acid and then stored at $-20^{\circ} \mathrm{C}$. Chromosome spreads were made by squashing fixed material onto a microscope slide in a drop of $45 \%(\mathrm{v} / \mathrm{v})$ acetic acid. Preparations with good chromosome morphology were immediately frozen by immersion in liquid nitrogen for $1 \mathrm{~min}$. After removing the coverslip, the slide was dehydrated through a graded ethanol series $(70 \%, 90 \%$ and $100 \%)$, then air dried.

\subsection{Probe labelling}

TAC15, a 30-kb positive clone containing the sequence of the Stpk- $V$ gene was obtained by Sun et al. [17]. TAC15 DNA was isolated by the QIAprep Spin Miniprep Kit (QIAGEN). TAC15 and $H$. villosa genomic DNA were labelled with digoxigenin-11-dUTP (Roche Diagnostics, Germany) by standard nick translation reactions, and purified by passage through Sephadex G-50.

\subsection{Sequential TAC-FISH and GISH}

FISH and sequential GISH were conducted essentially as 
described by Zhang et al. [25] and Dong et al. [26] with a few modifications. Chromosome-bearing slides were placed into $70 \%(\mathrm{v} / \mathrm{v})$ formamide in $2 \times \mathrm{SSC}$ and the chromosomes were denatured by heating at $88^{\circ} \mathrm{C}$ on a hot-plate for $3 \mathrm{~min}$ and immediately immersed in a graded ethanol series (70\%, $90 \%$ and $100 \%, 5$ min each) at $-20^{\circ} \mathrm{C}$. Total genomic DNA isolated from the leaves of T. aestivum cv. Chinese Spring and $H$. villosa were sheared to an average size of 100-300 bp by autoclaving at $120^{\circ} \mathrm{C}$ for $3 \mathrm{~min}$, then used as blocking DNA at a 300 -fold greater quantity than the probe. Digoxigenin-labelled probe was detected with FITC-conjugated anti-digoxigenin antibody (Roche Diagnostics, Germany). Chromosomes were counterstained with 4',6-diamidino-phenylindole (DAPI) in an anti-fade solution (Vector). Slides were examined under a fluorescence microscope (Olympus BX61, Japan). Chromosome and FISH signal images were captured using a charge-coupled device (CCD) camera and merged using IPLab Spectrum software (Signal Analytics, USA).

The slides were then soaked in phosphate-buffered saline (pH 7.4) to aid removal of the coverslips, the DNA was denatured again in $70 \%(\mathrm{v} / \mathrm{v})$ formamide at $80^{\circ} \mathrm{C}$ for $2 \mathrm{~min}$, and incubated with labelled $H$. villosa genomic DNA for sequential GISH. Chromosome images and GISH signals in the same metaphase cells selected in FISH analysis were captured and compared with the FISH results. The fraction length (FL) of hybridization sites was calculated as the distance from the centromere to the TAC-FISH signal relative to the total length of the chromosome arm. Measurements were made on digital images using IPLab Spectrum software. More than ten chromosomes were measured for each FL calculation and standard deviations were calculated.

\section{Results}

\subsection{Verification of materials by PCR amplification with the CINAU15 marker derived from the Stpk-V gene}

T. aestivum-H. villosa $6 \mathrm{~V}$ alien chromosome lines were developed in our earlier work and the PCR marker CINAU15 was designed on the basis of the sequence of the Stpk-V gene [13-15,19]. To further determine whether the genetic stocks used in this study conferred the Stpk- $V$ gene, PCR analysis with a pair of CINAU15 primers was done using the genomic DNA of $T$. aestivum cv. Chinese Spring, $T$. durum cv. 1286, H. villosa, T. durum-H. villosa amphiploid and $T$. aestivum $-H$. villosa $6 \mathrm{~V}$ alien chromosome lines. PCR products with a 902-bp band were detected in genomic DNA of these different genetic stocks containing the whole $6 \mathrm{VS}$ chromosome arm or the 6VS chromosome bin FL $0.00-0.58$, which were $H$. villosa, T. durum-H. villosa amphiploid and T. aestivum-H. villosa $6 \mathrm{~V}$ addition line 06R33, the $6 \mathrm{~V}(6 \mathrm{~A})$ substitution line $06 \mathrm{R} 41$, the $6 \mathrm{VS} / 6 \mathrm{AL}$ translocation line 92R90, 92R137 and its derivative variety Nannong 9918 and the deletion addition line del6VS-1 (Figure 1(a)). This 902-bp amplicon was not detected in T. aestivum cv. Chinese Spring, T. durum cv. 1286, the deletion addition line del6VS-2 that lacks the distal $55 \%$ of $6 \mathrm{VS}$, the $6 \mathrm{VS}$ small segment translocation lines HY86-1 and HY158-5 that contain FL0.00-0.45 and $0.70-1.00$ of the 6VS chromosome, respectively (Figure 1(a)). On the basis of these results of marker analysis, the $S t p k-V$ gene was located on the 6VS chromosome bin (FL: 0.45-0.58) (Figure 1(b)), and the genetic stocks harbouring the $S t p k-V$ gene, such as the $T$. aestivum $-H$. villosa $6 \mathrm{~V}$ addition line 06R33, the $6 \mathrm{~V}(6 \mathrm{~A})$ substitution line 06R41, the 6VS/6AL translocation lines 92R90 and 92R137, and its derivative variety Nannong 9918, and the deletion addition line del6VS-1 were suitable for subsequent cytogenetic analysis of this gene.

\subsection{Physical mapping of the Stpk- $V$ gene by sequential TAC-FISH and GISH in T. aestivum-H. villosa $6 \mathrm{~V}(6 \mathrm{~A})$} substitution, 6V addition and 6VS/6AL translocation lines

The T. aestivum-H. villosa $6 \mathrm{~V}(6 \mathrm{~A})$ substitution line 06R41 was used to determine the quantity of blocking genomic DNA in FISH with TAC15 as the probe. When the blocking DNA of T. aestivum cv. Chinese Spring and H. villosa were not used, painting-like signals were observed on a pair of $6 \mathrm{~V}$ chromosomes and some non-specific signals were detected on chromosomes of common wheat (Figure 2(a)). The result suggested that, in addition to the Stpk- $V$ gene, TAC15 contains repetitive sequence of $H$. villosa. when a 300-fold greater amount of blocking DNA of T. aestivum cv. Chinese Spring was added, all non-specific signals on the chromosomes of common wheat can be blocked and partial non-specific signals on the $6 \mathrm{~V}$ chromosomes of $H$. villosa are still detectable (Figure 2(b)). However, by adding a 300-fold greater amount of blocking DNA of both T. aestivum cv. Chinese Spring and H. villosa, non-specific noise on both $6 \mathrm{~V}$ chromosomes and the chromosomes of common wheat yielded by repetitive sequences of the TAC clone could be blocked, and clear double fluorescent signals were detected at both chromatids of a pair of chromosomes (Figure 2(c)), which were then determined to be a pair of $6 \mathrm{~V}$ chromosomes containing the $S t p k-V$ gene by a sequential GISH (Figure 2(d)). The TAC15 signals detected on the 6V chromosomes were analysed to determine the precise location of the Stpk-V gene, and the FL position of the Stpk-V locus was $0.587 \pm 0.040$ (Table 1 ).

TAC-FISH to mitotic metaphase chromosomes of T. aestivum-H. villosa addition and translocation lines were used to further confirm the physical location and to facilitate statistical analysis of the position of the gene, and similar discrete hybridization signals of Stpk-V loci were detected (Figure 3(a), (c), (e) and (g)). Following TAC-FISH, rehybridization of the chromosome preparations with $H$. villosa genomic DNA as the probe showed that chromosomes with strong hybridization signals were 6V or 6VS (Figure 3(b), (d), (f) and (h)). The FL position of the Stpk- $V$ locus 


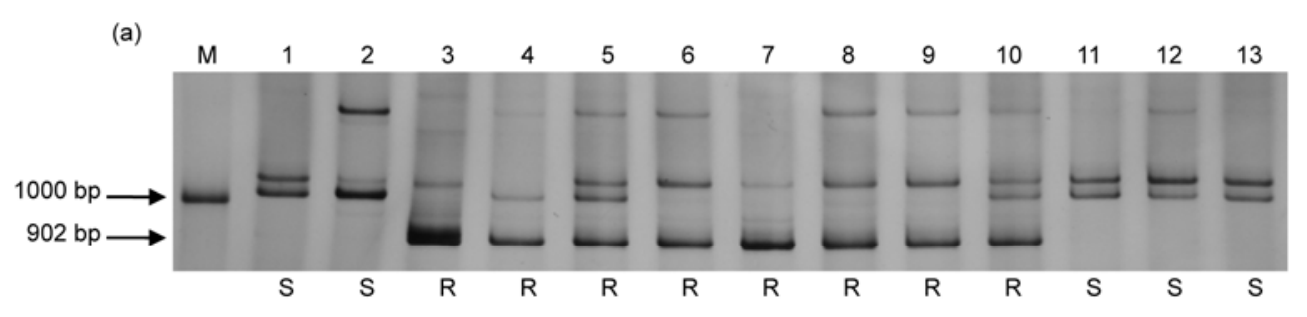

(b)

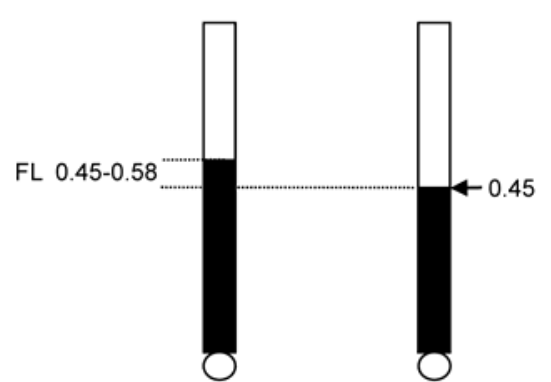

del 6VS-1 (R)

del 6VS-2 (S)

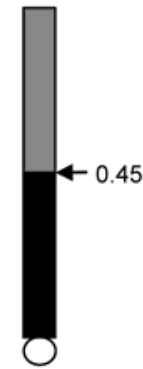

HY86-1 (S)

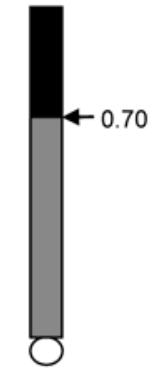

HY158-5 (S)

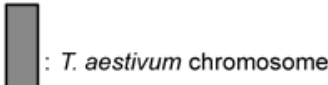

Figure 1 PCR analysis using a sequence-specific marker of the $S t p k-V$ gene in different genetic stocks located the $S t p k-V$ locus to the 6VS chromosome bin FL 0.45-0.58. (a) M, marker; 1, Chinese Spring; 2, T. durum cv. 1286; 3, H. villosa; 4, T. durum cv. 1286-H. villosa amphiploid; 5, T. aestivum-H. villosa 6V addition line 06R33; 6, T. aestivum-H. villosa 6V substitution line 06R41; 7, T. aestivum-H. villosa 6VS/6AL translocation line 92R137; 8, T. aestivum-H. villosa 6VS/6AL translocation line Nannong 9918; 9, T. aestivum-H. villosa 6VS/6AL translocation line 92R90; 10, T. aestivum-H. villosa 6VS deletion addition line del6VS-1; 11, T. aestivum-H. villosa 6VS deletion addition line del6VS-2; 12, T. aestivum-H. villosa 6VS small segment translocation line HY158-5; 13, T. aestivum-H. villosa 6VS small segment translocation line HY86-1. R: resistance to powdery mildew; S: susceptible to powdery mildew. (b) A diagram of 6VS or 6VS segment in different genetic stocks.
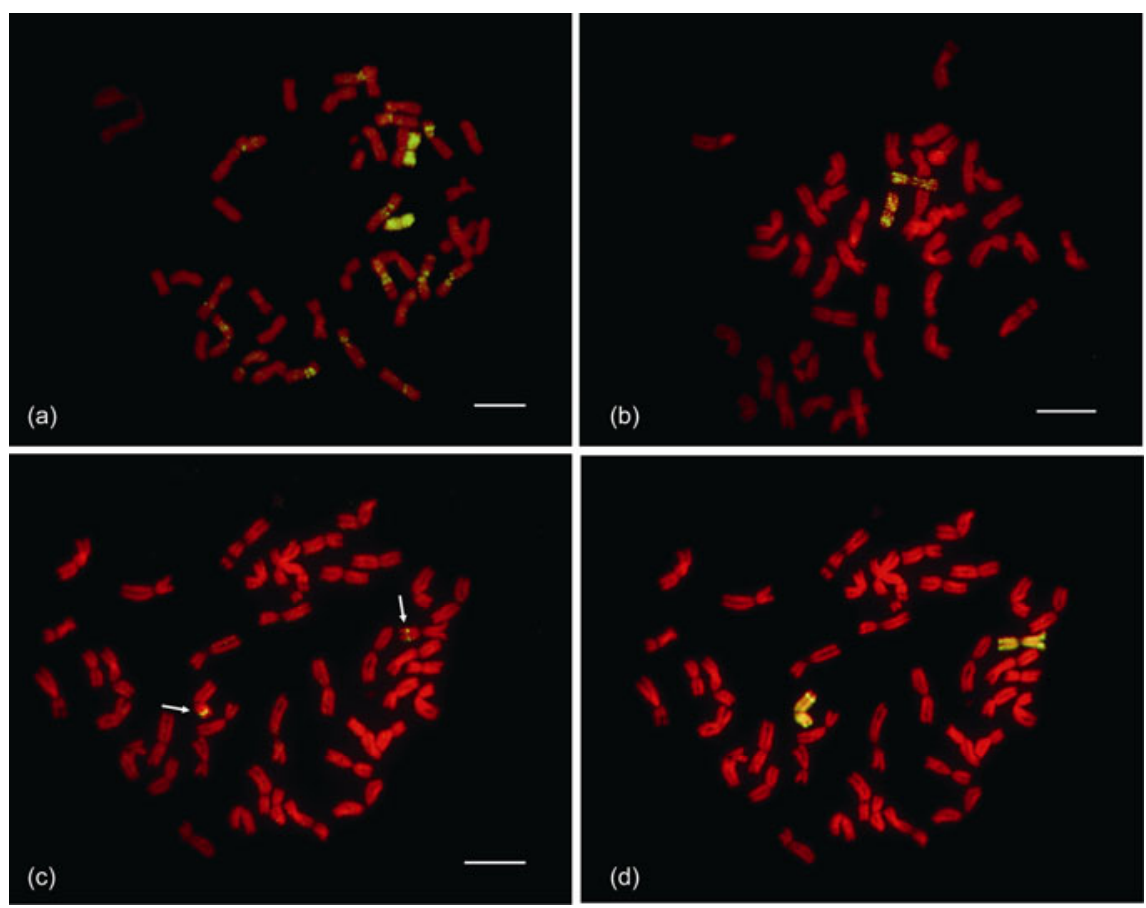

Figure 2 FISH with TAC15 as the probe on metaphase chromosomes of T. aestivum-H. villosa $6 \mathrm{~V}$ substitution line 06R41. Hybridization signals were detected with FITC-anti-digoxigenin, and chromosomes were counterstained with DAPI. Chromosomes are coloured red. The scale bars represent $10 \mu \mathrm{m}$. (a) FISH with TAC15 as the probe on metaphase chromosomes without adding blocking DNA of Chinese Spring and $H$. villosa. (b) FISH with TAC15 as the probe on metaphase chromosomes by adding 300-fold blocking DNA of Chinese Spring. (c) FISH with TAC15 as the probe on metaphase chromosomes by adding 300-fold blocking DNA of both Chinese Spring and H. villosa. Arrows indicate the signals on both chromatids of chromosome 6V. (d) Sequential GISH with the genomic DNA of $H$. villosa as the probe. 


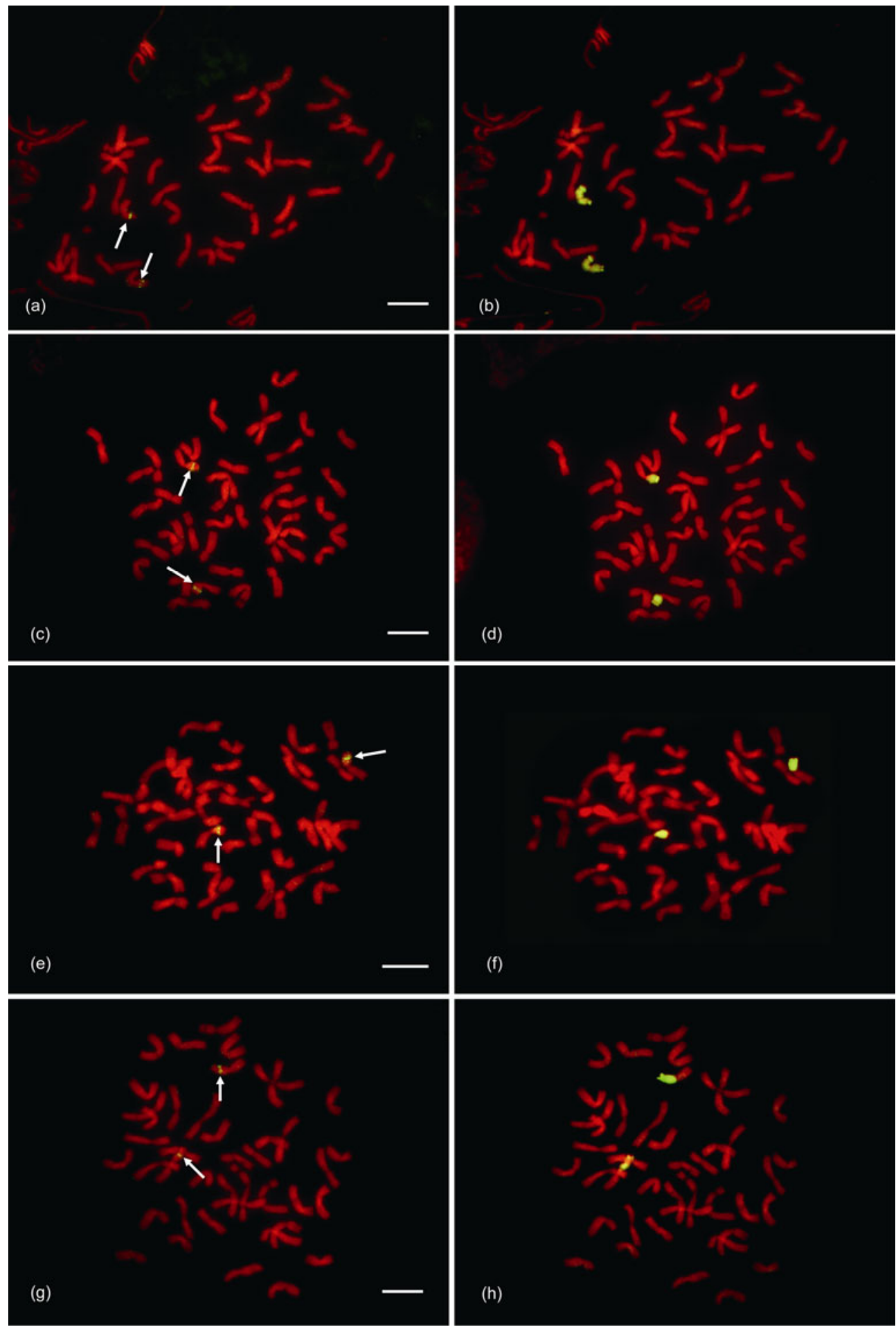

Figure 3 FISH with TAC15 as the probe (a, c, e and g) and GISH with the genomic DNA of $H$. villosa as the probe (b, d, f and h) hybridized to cells with complete mitotic metaphase chromosomes of different genetic stocks containing 6V. Hybridization signals were detected with FITC-anti-digoxigenin, and chromosomes were counterstained with DAPI. Chromosomes are coloured red. Arrows indicate the signals on both chromatids. The scale bars represent $10 \mu \mathrm{m}$. (a) and (b) T. aestivum-H. villosa 6V addition line 06R33; (c) and (d) T. aestivum-H. villosa 6VS/6AL translocation line 92R137; (e) and (f) T. aestivum-H. villosa 6VS/6AL translocation line Nannong 9918; (g) and (h) T. aestivum-H. villosa 6VS/6AL translocation line 92R90.

was $0.573 \pm 0.033,0.566 \pm 0.034,0.574 \pm 0.039$ and $0.578 \pm$ 0.021 in $T$. aestivum $-H$. villosa $6 \mathrm{~V}$ addition line 06R33, 6VS/6AL translocation line 92R137, Nannong 9918 and
92R90, respectively. In summary, the FL position of the Stpk-V locus was found to be $0.575 \pm 0.035$ on the $6 \mathrm{~V}$ chromosome short arm (Table 1). 
Table 1 Physical location of TAC15 in different genetic stocks containing $6 \mathrm{~V}$ chromosome

\begin{tabular}{llcc}
\hline Genotype & Type & $\begin{array}{c}\text { Number of } \\
\text { chromosomes measured }\end{array}$ & $\begin{array}{c}\text { FL value and } \\
\text { standard deviation }\end{array}$ \\
\hline 06R33 & Add 6V & 13 & $0.573 \pm 0.033$ \\
06R41 & Sub 6V & 21 & $0.587 \pm 0.040$ \\
92R137 & 6VS/6AL & 27 & $0.566 \pm 0.034$ \\
Nannong 9918 & 6VS/6AL & 20 & $0.574 \pm 0.039$ \\
92R90 & 6VS/6AL & 20 & $0.578 \pm 0.021$ \\
\hline Total & & 101 & $0.575 \pm 0.035$ \\
\hline
\end{tabular}

2.3 Tracing the location of the Stpk- $V$ gene by sequential TAC-FISH in $T$. aestivum-H. villosa $6 \mathrm{~V}$ alien chromosome lines

FISH with TAC15 as the probe was used for tracing the location of the Stpk- $V$ gene in different genotypes containing the 6VS chromosome segment. Discrete hybridization signals of Stpk- $V$ loci were not detected in genotypes susceptible to powdery mildew, such as the alien deletion addition line del6VS-2 (FL 0.45) and the 6VS small segment translocation lines HY86-1 and HY158-5, which contain the 6VS chromosome bin at FL0.00-0.45 and 0.70-1.00, respectively (data not shown). FISH signals of the Stpk- $V$ locus were detected in the deletion addition line del6VS-1 (FL 0.58 ) and the 6VS small translocation lines YC72-2 and YC138-4, which are resistant to powdery mildew. Figure 4(a) shows that the fluorescence signals occurring at the terminal region of a pair of chromosomes in deletion addition line del6VS-1 and chromosomes with fluorescent signals were identified as a pair of $H$. villosa chromosomes by sequential GISH with $H$. villosa genomic DNA as the probe (Figure 4(b)). Figure 4(c) and (e) show the clear doublet signals of FISH with TAC15 as the probe in the 6VS small
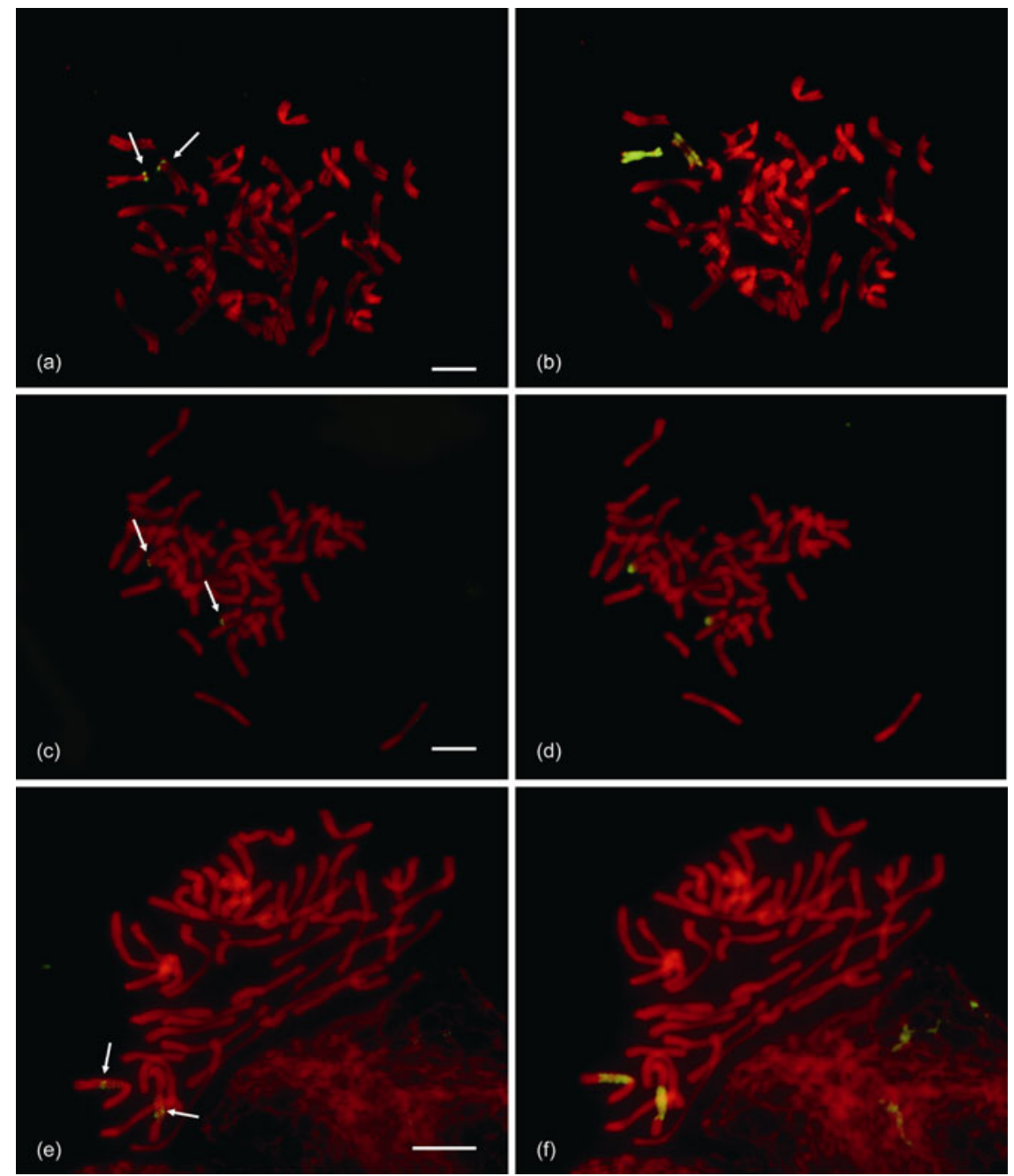

Figure 4 Sequential FISH with TAC15 as the probe (a, c and e) and GISH with the genomic DNA of $H$. villos $a$ as the probe (b, d and f) for tracing the location of the Stpk-V gene in T. aestivum-H. villosa $6 \mathrm{~V}$ alien chromosome lines. Hybridization signals were detected with FITC-anti-digoxigenin and chromosomes were counterstained with DAPI. Chromosomes are coloured red. Arrows indicate the signals on both chromatids. The scale bars represent $10 \mu \mathrm{m}$. (a) and (b) T. aestivum-H. villosa 6V deletion addition line del6VS-1; (c) and (d) T. aestivum-H. villosa 6VS small segment translocation line YC72-2; (e) and (f) T. aestivum-H. villosa 6VS small segment translocation line YC138-4. 
segment translocation lines YC72-2 and YC138-4. Sequential GISH with the genomic DNA of $H$. villosa as the probe showed the chromosome segment carrying the $\operatorname{Stpk}-V$ gene was from the $H$. villosa chromosome (Figure 4(d) and (f)).

\section{Discussion}

In plants, many BACs or YACs carrying single- or lowcopy sequences contain repetitive sequences that hamper detection of the single- or low-copy sequences by FISH and FISH of most plant BACs results in strong background signals from multi-site hybridization of repeated sequences. Competitive in situ suppression with blocking DNA, using the unlabelled Cot-1 fraction or sheared genomic DNA, is of significant help for mapping genes by BAC- or YACFISH of some plant species with relatively small genomes. In rice, the bacterial blight disease-resistance genes Xa21 and $x a 5$, the leaf blast-resistant genes $P i-b$ and $P i-5(t)$ and the gall midge resistance gene $G m 2$ were physically mapped to metaphase chromosomes by BAC-FISH or YAC-FISH [5-9]. However, BAC-FISH for physical mapping in plants possessing large genomes, such as wheat and barley, might be questionable $[3,27]$. Most of the BAC clones hybridized as dispersed signals on all chromosomes and it was not easy to obtain a discrete signal even when a large excess of suppressor DNA was added. Owing to the large proportion $(>80 \%)$ of repetitive sequences in genome of wheat, it is extremely difficult to generate unique locus-specific FISH signals for most wheat BACs $[3,28]$. In wheat, there were only a few reports about successfully mapping using BAC clones, such as the BACs screened from the Aegilops tauschii and T. monococcum could hybridized to specific chromosomes of wheat [3].

BACs or TACs with low-copy repetitive sequences were obtained in our laboratory by screening a wheat BAC or TAC library with molecular markers. Only a few BACs or TACs yielding discrete signals were identified by FISH. In this study, the quantity and type of blocking DNAs is important to suppress the background signals generated by the repetitive sequence of TAC15. When the quantity of blocking DNA was 300-fold greater compared to the amount of labelled probe TAC15, either genomic DNA from $T$. aestivum cv. Chinese Spring or from H. villosa could not suppress the background signals completely. Ratios for sheared genomic DNA to labelled probe ranged from 50 to 150 -fold were not able to detect distinctive signals (data not shown). Specific hybridization signals generated by TAC15 are clearly distinguishable only when using a $>300$-fold greater amount of sheared genomic DNA of both T. aestivum cv. Chinese Spring and $H$. villosa compared to labelled TAC15. The signals were detected on both chromatids using different T. aestivum $-H$. villosa $6 \mathrm{~V}$ alien chromosome lines, indicating that the signals obtained by FISH were genuine and the results of FISH mapping were reliable. After detecting $>100$ chromosomes of 6VS with TAC15 signals, the Stpk-V locus was physically located at the position of 6VS FL $0.575 \pm$ 0.035 .

In our earlier work, when labelled TAC15 probe was hybridized to the metaphase chromosomes of $H$. villosa, background signals produced by repetitive sequences of TAC15 were dispersed over all chromosomes and failed to suppress the background signals, even by using blocking DNA of both $T$. aestivum cv. Chinese Spring and H. villosa. The FISH signals of TAC15 containing Stpk-V loci were more easily visible with minimal interference of the $\mathrm{V}$-genome in T. aestivum-H. villosa alien chromosome lines with a pair of $\mathrm{V}$-genome chromosome than in $H$. villosa with the whole $\mathrm{V}$ genome. Therefore, the use of T. aestivum $-H$. villosa $6 \mathrm{~V}$ alien chromosome lines and genomic DNA of both T. aestivum cv. Chinese Spring and $H$. villosa for blocking are important for mapping the $S t p k-V$ gene. The gene physical mapping strategies used in this study will be helpful for tracing alien genes from wild species in T. aestivum.

This work was supported by the National Basic Research Program of China (2009CB118304), the National Natural Science Foundation of China (31171540, 30871519), the Program for New Century Excellent Talents in University (NCET-10-0496), the Independent Innovation of Agricultural Sciences $(C X(11) 1025)$ and the Sci \& Tech Project in Jiangsu Province (BE2011306).

1 Jiang J, Gill B S. Current status and the future of fluorescence in situ hybridization (FISH) in plant genome research. Genome, 2006, 49: 1057-1068

2 Raina S N, Rani V. GISH technology in plant genome research. Methods Cell Sci, 2001, 23: 83-104

3 Zhang P, Li W, Fellers J, et al. BAC-FISH in wheat identifies chromosome landmarks consisting of different types of transposable elements. Chromosoma, 2004, 112: 288-299

4 Friebe B, Jiang J, Raupp W J, et al. Characterization of wheat-alien translocations conferring resistance to diseases and pests: Current status. Euphytica, 1996, 91: 59-87

5 Jiang J, Gill B S, Wang G, et al. Metaphase and interphase fluorescence in situ hybridization mapping the rice genome with bacterial artificial chromosomes. Proc Natl Acad Sci USA, 1995, 92: 44874491

6 Ohmido N, Akiyama Y, Fukui K. Physical mapping of unique nucleotide sequences on identified rice chromosomes. Plant Mol Biol, 1998, 18: 1043-1052

7 Rajyashri K R, Nair S, Ohmido N, et al. Isolation and FISH mapping of Yeast Artificial Chromosomes (YACs) encompassing an allele of the $G m 2$ gene for gall midge resistance in rice. Theor Appl Genet, 1998, 97: 507-514

8 Qin R, Wei W, Jin W, et al. Physical location of rice Gm-6, Pi-5(t) genes in O. officinalis with BAC-FISH. Chin Sci Bull, 2001, 46: 659-661

9 Xiong Z, Tan G, You A, et al. Comparative physical mapping of rice BAC clones linked to resistance gene Glh, Bph-3 and xa-5 in Oryza sativa L. and O. granulata Nees et Arn. Ex Watt. Chin Sci Bull, 2004, 49: 591-596

10 Gómez M I, Islam-Faridi M N, Woo S, et al. FISH of a maize sh2-selected sorghum BAC to chromosomes of Sorghum bicolor. Genome, 1997, 40: 475-478

11 Suzuki G, Tanaka S, Yamamoto M, et al. Visualization of the $S$ locus region in Ipomoea trifida: Toward positional cloning of self-incompatibility genes. Chromosome Res, 2004, 12: 475-481 
12 Desel C, Jung C, Cai D, et al. High-resolution mapping of YACs and the single-copy gene $H_{s} 1^{\text {pro-l }}$ on Beta vulgaris chromosomes by multicolor fluorescence in situ hybridization. Plant Mol Biol, 2001, 45: 113-122

13 Chen P D, Qi L L, Zhou B, et al. Development and molecular cytogenetic analysis of wheat-Haynaldia villosa $6 \mathrm{VS} / 6 \mathrm{AV}$ translocation lines specifying resistance to powdery mildew. Theor Appl Genet, 1995, 91: 1125-1128

14 Qi L L, Wang S L, Chen P D, et al. Identification and physical mapping of three Haynaldia villosa chromosome-6V deletion lines. Theor Appl Genet, 1998, 97: 1042-1046

15 Cao A Z, Wang X E, Chen Y P, et al. A sequene-specific PCR marker linked with Pm21 distinguishes chromosomes 6AS, 6BS, 6DS of Triticum aestivum and 6VS of Haynaldia villosa. Plant Breed, 2006, 125: 201-205

16 Fan Y D, Liu Y G, Wu H, et al. Construction of a transformationcompetent artificial chromosome (TAC) library of a wheatHaynaldia villosa translocation line (in Chinese). Chin J Biotech, 2000, 16: 433-436

17 Sun Y L, Cao A Z, Yang X M, et al. Screening $H v-S / T P K$ from TAC library of a Triticum aestivum-Haynaldia villosa translocation line (in Chinese). Chin J Biotech, 2008, 24: 1327-1332

18 Cao A, Xing L, Wang X, et al. Serine/threonine kinase gene Stpk-V, a key member of powdery mildew resistance gene Pm21, confers powdery mildew resistance in wheat. Proc Natl Acad Sci USA, 2011, 108: 7727-7732

19 Chen P D, Liu D J. Identification of Haynaldia villosa chromosomes in alien wheat alien addition lines. In: Li Z S, Swaminathan M S, eds. Proc 1st Int Symp on Chromosome Engineering in Plants, Xi'an,
China, 1986. 31-33

20 Pei G Z, Chen P D, Liu D J. A cytogenetic analysis of some powdery mildew resistant strains of the hybrid progeny between wheat and Haynaldia villosa (in Chinese). J Nanjing Agric Univ, 1986, 1: 1-9

21 Liu D J, Chert P D, Pei G Z, et al. Transfer of Haynaldia villosa chromosomes into Triticum aestivum. In: Miller T E, Koebner R M D, eds. Proc 7th Int Wheat Genet Symp, Cambridge, UK, 1988. 355361

22 Chen P D, Zhang S Z, Wang X E, et al. New wheat variety Nannong 9918 with high yield and powdery mildew resistance (in Chinese). J Nanjing Agric Univ, 2002, 25: 105-106

23 Chen S, Chen P, Wang X. Inducement of chromosome translocation with small alien segments by irradiating mature female gametes of the whole arm translocation line. Sci China Ser C Life Sci, 2008, 51: 346-352

24 Qi L L, Wang S L, Chen P D, et al. Molecular cytogenetic analysis of Leymus racemosus chromosomes added to wheat. Theor Appl Genet, 1997, 95: 1084-1091

25 Zhang D, Yang Q, Bao W, et al. Molecular cytogenetic characterization of the Antirrhinum majus genome. Genetics, 2005, 169: 325-335

26 Dong F, McGrath J M, Helgeson J P, et al. The genetic identity of alien chromosomes in potato breeding lines revealed by sequential GISH and FISH analyses using chromosome-specific cytogenetic DNA markers. Genome, 2001, 44: 729-734

27 Lengerova M, Kejnovsky E, Hobza R, et al. Multicolor FISH mapping of the dioecious model plant, Silene latifolia. Theor Appl Genet, 2004, 108: 1193-1199

28 Smith D B, Flavell R B. Characterization of the wheat genome by renaturation kinetics. Chromosoma, 1975, 50: 223-242

Open Access This article is distributed under the terms of the Creative Commons Attribution License which permits any use, distribution, and reproduction in any medium, provided the original author(s) and source are credited. 